\title{
QUEEN'S
UNIVERSITY
BELFAST
}

\section{Physical and numerical constraints in source modeling for finite difference simulation of room acoustics}

Sheaffer, J., Walstijn, M. V., \& Fazenda, B. (2014). Physical and numerical constraints in source modeling for finite difference simulation of room acoustics. The Journal of the Acoustical Society of America, 135(1), $251-261$. https://doi.org/10.1121/1.4836355

\section{Published in:}

The Journal of the Acoustical Society of America

\section{Document Version:}

Peer reviewed version

Queen's University Belfast - Research Portal:

Link to publication record in Queen's University Belfast Research Portal

\section{Publisher rights}

(c) 2014 Acoustical Society of America.

This article may be downloaded for personal use only. Any other use requires prior permission of the author and the Acoustical Society of America.

The following article appeared in J. Acoust. Soc. Am. 135, 251 (2014); and may be found at http://dx.doi.org/10.1121/1.4836355.

\section{General rights}

Copyright for the publications made accessible via the Queen's University Belfast Research Portal is retained by the author(s) and / or other copyright owners and it is a condition of accessing these publications that users recognise and abide by the legal requirements associated with these rights.

Take down policy

The Research Portal is Queen's institutional repository that provides access to Queen's research output. Every effort has been made to ensure that content in the Research Portal does not infringe any person's rights, or applicable UK laws. If you discover content in the Research Portal that you believe breaches copyright or violates any law, please contact openaccess@qub.ac.uk. 


\title{
Physical and Numerical Constraints in Source Modeling for Finite Difference Simulation of Room Acoustics ${ }^{\text {a) }}$
}

\author{
Jonathan Sheaffer ${ }^{\mathrm{b})}$ \\ School of Computing, \\ Science and Engineering, \\ University of Salford, \\ $U K$ \\ Maarten van Walstijn \\ School of Electronics, \\ Electrical Engineering and Computer Science. Queen's University Belfast, \\ $U K$ \\ Bruno Fazenda \\ School of Computing, \\ Science and Engineering, \\ University of Salford. UK
}

(Dated: July 9, 2013)

a) Portions of this work were presented in: A physically-constrained source model for FDTD acoustic simulation, Proc. of the 15th Int. Conference on Digital Audio Effects (DAFx12), York UK, September 2012 


\begin{abstract}
In finite difference time domain simulation of room acoustics, source functions are subject to various constraints. These depend on the way sources are injected into the grid and on the chosen parameters of the numerical scheme being used. This paper addresses the issue of selecting and designing sources for finite difference simulation, by first reviewing associated aims and constraints, and evaluating existing source models against these criteria. The process of exciting a model is generalized by introducing a system of three cascaded filters respectively characterizing the driving pulse, the source mechanics, and the injection of the resulting source function into the grid. It is shown that hard, soft and transparent sources can be seen as special cases within this unified approach. Starting from the mechanics of a small pulsating sphere, a parametric source model is formulated by specifying suitable filters. This physically constrained source model is numerically consistent, does not scatter incoming waves and is free from DC and low-frequency artifacts. Simulation results are employed for comparison with existing source formulations in terms of meeting the spectral and temporal requirements on the outward propagating wave.
\end{abstract}

PACS numbers: 43.55.Ka,43.38.Ar,43.55.Lb 


\section{INTRODUCTION}

The Finite Difference Time Domain (FDTD) method has recently gained in applicability to room acoustics, largely owing to improved boundary formulations ${ }^{1-4}$, newly emerged schemes $^{5,6}$, and hardware-accelerated implementations ${ }^{7-9}$. Among the various FDTD modeling aspects, grid excitation has received relatively sparse attention in the literature, with researchers in acoustics usually directly employing the methods inherited from their counterparts in the field of electromagnetics.

In FDTD simulation of electromagnetic fields, where the numerical scheme approximates a solution to Maxwell's equations ${ }^{10}$, a general distinction is made between a Hard Source (HS), which imposes a voltage or current on the electrical field, and a Soft Source (SS), which superimposes either variable onto the field ${ }^{11,12}$. By analogy, these forms of injecting energy into the grid can be used to simulate pressure and velocity sources in an acoustic field.

While in the first acoustic FDTD formulation by Botteldooren ${ }^{13}$ the field was excited by imposing velocity across an area representing a speaker membrane, subsequent acoustic studies have often made use of omni-directional sources via HS or SS excitation at a single grid node. Similar source formulations can be found in the closely related simulation paradigm of digital waveguide modeling ${ }^{14,15}$.

One advantage of HS over SS excitation is that it allows a more precise control of the outward propagating pressure wave, which facilitates various modeling aims, such as field visualization and response analysis ${ }^{16}$. However, unlike with soft sources, waves propagating back to the source reflect from a hard source node ${ }^{17}$, effectively imposing a severe limit on the available time window. Schneider and colleagues ${ }^{18,19}$ addressed this major drawback by proposing Transparent Sources (TS), which generate the same pressure field as a HS but avoid the source node scattering by means of reflection cancellation; this involves measur-

b)URL: http://www.acoustics.salford.ac.uk/res/; Electronic address: j.sheaffer@ edu.salford.ac.uk 
ing the grid impulse response prior to the principal numerical experiment, which carries a significant additional computational effort. A similarity between TS and the so-called total-field/scattered field and pure scattered field formulations was noted by Redondo and colleagues $^{20}$.

More recently, Jeong and Lam ${ }^{21}$ showed that HS and TS are prone to undesired lowfrequency artifacts when certain excitation functions are used, and proposed the use of sinemodulated Gaussian pulses - which are not spectrally flat - to address this. In a similar vein, differentiated pulses have been in use in electromagnetic FDTD for some time, in order to avoid DC excitation ${ }^{11,12}$. These solutions exemplify the inherent trade-offs in FDTD source modeling, in this case balancing the elimination of low-frequency artifacts with effecting an outward wave of desirable frequency content. These findings also suggest that the methods for shaping and for injection of the source pulse should not be seen and chosen in isolation. The literature does, however, not give a clear view of how the various criteria relate to the underlying physics and the employed numerical formulations.

In order to obtain a broader insight into how trade-offs can be made in the design of acoustic FDTD source models, this paper addresses the problem by first reviewing the associated aims and constraints. Several methods for injection and pulse shaping are then evaluated against these criteria (Section III). In the following section, grid excitation modeling is generalized in the form of a digital filter chain, each filter representing a separate constraining system; this processing structure converts an arbitrarily chosen excitation signal into a final source function. Starting from a small pulsating sphere model, a new excitation method is then formulated by specifying suitable filters. Finally, the resulting PhysicallyConstrained Source (PCS) model is evaluated through numerical results and compared to existing methods in Section V. 


\section{THE FDTD METHOD IN ACOUSTICS}

\section{A. Yee-type Method}

The original FDTD method for electrodynamics suggested by Yee $^{10}$ makes use of two staggered grids representing the electric and magnetic fields. In the field of acoustics, the method was adapted to solve Euler's linearized equations ${ }^{13}$, which represent propagation of pressure and particle velocity, and will be further referred to as a Yee-type method. When sources are present in the domain, the conservation laws of mass and momentum describing the sound field at $\mathbf{x}=(x, y, z) \in \mathbb{R}^{3}$, are given by $^{22}$

$$
\begin{gathered}
\frac{1}{c^{2}} \frac{\partial p(\mathbf{x}, t)}{\partial t}+\rho_{0} \nabla \cdot \mathbf{u}(\mathbf{x}, t)=q(\mathbf{x}, t) \\
\rho_{0} \frac{\partial \mathbf{u}(\mathbf{x}, t)}{\partial t}+\nabla p(\mathbf{x}, t)=\widetilde{\mathbf{F}}(\mathbf{x}, t)
\end{gathered}
$$

where $p(\mathbf{x}, t)$ is sound pressure, $\mathbf{u}(\mathbf{x}, t)$ is particle velocity, $\rho_{0}$ is the ambient density of air and $c$ is the velocity of sound in air. Here, the function $q(\mathbf{x}, t)$ denotes the rate of fluid emergence in the system in the dimension of density per unit time $\left(\mathrm{Kg} \mathrm{m}^{-3} \mathrm{~s}^{-1}\right)$, and the function $\widetilde{\mathbf{F}}(\mathbf{x}, t)$ is the acoustic force exerted upon the source volume. For simplicity, it is assumed that all considered excitation functions represent volume velocity sources, and as such, the force term in Equation (2), is neglected. Accordingly, Equations (1), and (2), can be approximated using finite difference operators as

$$
\left.\delta_{t} p\right|_{\mathbf{i}} ^{n}=\underbrace{\left.c^{2} T q\right|_{\mathbf{i}} ^{n}}_{\text {Source Term }}-z_{0} \lambda\left(\left.\delta_{x} u_{x}\right|_{\mathbf{i}} ^{n}+\left.\delta_{y} u_{y}\right|_{\mathbf{i}} ^{n}+\left.\delta_{z} u_{z}\right|_{\mathbf{i}} ^{n}\right)
$$

and

$$
\begin{aligned}
& \left.\delta_{t} u_{x}\right|_{\mathbf{i}} ^{n}=-\left.\frac{\lambda}{z_{0}} \delta_{x} p\right|_{\mathbf{i}} ^{n} \\
& \left.\delta_{t} u_{y}\right|_{\mathbf{i}} ^{n}=-\left.\frac{\lambda}{z_{0}} \delta_{y} p\right|_{\mathbf{i}} ^{n} \\
& \left.\delta_{t} u_{z}\right|_{\mathbf{i}} ^{n}=-\left.\frac{\lambda}{z_{0}} \delta_{z} p\right|_{\mathbf{i}} ^{n}
\end{aligned}
$$

where $u_{x}, u_{y}$ and $u_{z}$ denote the orthogonal components of the particle velocity vector $\mathbf{u}$ in a Cartesian coordinate system, $z_{0}=\rho_{0} c$ is the characteristic impedance of air, and 
$\lambda=c T / X$ is the Courant number ${ }^{23}$. In the numerical domain, the system is sampled such that $(x, y, z, t) \rightarrow[l X, m X, i X, n T]$ and accordingly $n$ and $\mathbf{i}=[l, m, i]$ are the index positions in discrete time and space, and $X$ and $T$ are respectively the spatial and temporal sample periods. The finite difference operators are given by

$$
\begin{aligned}
\left.\left.\delta_{t} \mathbf{u}\right|_{\mathbf{i}} ^{n} \equiv \mathbf{u}\right|_{\mathbf{i}} ^{n+\frac{1}{2}}-\left.\mathbf{u}\right|_{\mathbf{i}} ^{n-\frac{1}{2}} & \left.\left.\delta_{t} p\right|_{\mathbf{i}} ^{n} \equiv p\right|_{\mathbf{i}} ^{n+1}-\left.p\right|_{\mathbf{i}} ^{n} \\
\left.\left.\delta_{x} \mathbf{u}\right|_{\mathbf{i}} ^{n} \equiv \mathbf{u}\right|_{l+\frac{1}{2}, m, i} ^{n+\frac{1}{2}}-\left.\mathbf{u}\right|_{l-\frac{1}{2}, m, i} ^{n+\frac{1}{2}} & \left.\left.\delta_{x} p\right|_{\mathbf{i}} ^{n} \equiv p\right|_{l+1, m, i} ^{n}-\left.p\right|_{l, m, i} ^{n} \\
\left.\left.\delta_{y} \mathbf{u}\right|_{\mathbf{i}} ^{n} \equiv \mathbf{u}\right|_{l, m+\frac{1}{2}, i} ^{n+\frac{1}{2}}-\left.\mathbf{u}\right|_{l, m-\frac{1}{2}, i} ^{n+\frac{1}{2}} & \left.\left.\delta_{y} p\right|_{\mathbf{i}} ^{n} \equiv p\right|_{l, m+1, i} ^{n}-\left.p\right|_{l, m, i} ^{n} \\
\left.\left.\delta_{z} \mathbf{u}\right|_{\mathbf{i}} ^{n} \equiv \mathbf{u}\right|_{l, m, i+\frac{1}{2}} ^{n+\frac{1}{2}}-\left.\mathbf{u}\right|_{l, m, i-\frac{1}{2}} ^{n+\frac{1}{2}} & \left.\left.\delta_{y} p\right|_{\mathbf{i}} ^{n} \equiv p\right|_{l, m, i+1} ^{n}-\left.p\right|_{l, m, i} ^{n}
\end{aligned}
$$

By direct substitution of (5) into (3) and (4), and by removing any source terms, the update equations for air are obtained, as originally formulated by Botteldooren ${ }^{13}$.

\section{B. Scalar Wave Equation Method}

While the Yee scheme is a popular choice of many authors, it is by no means the most efficient solution for room acoustics simulation ${ }^{24}$. In fact, if knowledge of particle velocity is not required throughout the entire soundfield, then one may employ a finite difference scheme approximating the scalar wave equation for pressure, a formulation which is here referred to as the Wave Equation Method ${ }^{5}$. Accordingly, when sources are present in the domain, one considers the inhomogeneous wave equation,

$$
\frac{1}{c^{2}} \frac{\partial^{2} p(\mathbf{x}, t)}{\partial t^{2}}-\nabla^{2} p(\mathbf{x}, t)=\psi(\mathbf{x}, t)
$$

To enable a direct comparison with other studies, here $\psi(\mathbf{x}, t)$ is defined as a general source driving function, whose physical relation to fluid emergence in the system shall be further discussed in Section III. Using the same nomenclature, the wave equation can be discretized as

$$
\left.\left(\delta_{t}^{2}-\lambda^{2} \delta_{\mathbf{x}}^{2}\right) p\right|_{\mathbf{i}} ^{n}=\underbrace{\left.c^{2} T^{2} \psi\right|_{\mathbf{i}} ^{n}}_{\text {Source Term }}
$$


with the finite difference operators given as

$$
\begin{gathered}
\left.\left.\delta_{t}^{2} p\right|_{\mathbf{i}} ^{n} \equiv p\right|_{\mathbf{i}} ^{n+1}-\left.2 p\right|_{\mathbf{i}} ^{n}+\left.p\right|_{\mathbf{i}} ^{n-1} \\
\left.\left.\delta_{x}^{2} p\right|_{\mathbf{i}} ^{n} \equiv p\right|_{l+1, m, i} ^{n}-\left.2 p\right|_{l, m, i} ^{n}+\left.p\right|_{l-1, m, i} ^{n} \\
\left.\left.\delta_{y}^{2} p\right|_{\mathbf{i}} ^{n} \equiv p\right|_{l, m+1, i} ^{n}-\left.2 p\right|_{l, m, i} ^{n}+\left.p\right|_{l, m-1, i} ^{n} \\
\left.\left.\delta_{z}^{2} p\right|_{\mathbf{i}} ^{n} \equiv p\right|_{l, m, i+1} ^{n}-\left.2 p\right|_{l, m, i} ^{n}+\left.p\right|_{l, m, i-1} ^{n}
\end{gathered}
$$

where the operator $\delta_{\mathbf{x}}^{2}$ is given by

$$
\delta_{\mathbf{x}}^{2}=\delta_{x}^{2}+\delta_{y}^{2}+\delta_{z}^{2}+a\left(\delta_{x}^{2} \delta_{y}^{2}+\delta_{x}^{2} \delta_{z}^{2}+\delta_{y}^{2} \delta_{z}^{2}\right)+b \delta_{x}^{2} \delta_{y}^{2} \delta_{z}^{2}
$$

The free parameters $a$ and $b$ are chosen according to the desired properties of the numerical scheme being used. By setting $a=0, b=0$, applying the finite difference operators to Equation (7), and removing the source term, one obtains the well known update equation for air in a rectilinear node arrangement ${ }^{5}$.

\section{SOURCE MODELING REVIEW}

\section{A. General Aims}

In order to assess the merits and shortcomings of existing source models, it is useful to review some of the requirements for an idealized sound source in room acoustics simulation, which are generally similar to those of an acoustic measurement. First, it is desired that the bandwidth of the source is wide enough to cover the entire frequency range of interest, and that it is sufficiently flat within that range ${ }^{25,26}$. The sound source should generate a prescribed pressure field, meaning that one should be able to predict its magnitude in free field. In many cases, it is useful to have a source that can excite the room omni-directionally at all frequencies of interest ${ }^{27}$ (at least within the dispersion limitations of the numerical scheme). It is also important that the process of grid excitation is numerically consistent, meaning that a change in grid parameters would not affect the magnitude of the sound field generated by the source. Also, when transient phenomena are investigated, it is desired that 
the source excitation signal is sufficiently compact in time, so that temporal overlap between discrete reflections is minimized. Lastly, although never feasible in a physical measurement, it is useful to be able to excite the room transparently, that is, without introducing scattering effects from the source itself.

\section{B. Physical Constraints}

Equation (1), relates the time derivative of pressure and space derivatives of particle velocity to the rate of fluid emergence, $q(\mathbf{x}, t)$, which shall now be developed mathematically. In acoustics, a fundamental type of source known as a point monopole is a limitingly small object which radiates spherical wavefronts ${ }^{28}$. Radiation could be caused, for example, due to a time-varying heat, or some mechanical force causing a sphere to pulsate and generate a volumetric flow (such a system will be described in more detail in Section IV.A). In the limiting case, where the physical size of the object approaches zero, the soundfield at the source position, $\mathbf{x}^{\prime}=\left(x^{\prime}, y^{\prime}, z^{\prime}\right) \in \mathbb{R}^{3}$, approaches a point of singularity in which the homogeneous wave equation is not satisfied. The rate of fluid emergence inside a small volume $V$ surrounding this point source must equal the local mass flow rate divided by $V$ :

$$
q(\mathbf{x}, t)=\frac{\rho_{0} Q(t) \delta\left(\mathbf{x}-\mathbf{x}^{\prime}\right)}{V}
$$

where $Q(t)$ is the volumetric flow rate, or volume velocity of the source. In anticipation of how this applies to a discretized system in which $V$ is the volume occupied by a single FDTD node, it can be seen that Equation (13), changes the dimension of volume velocity and, as such, presents a scaling constraint relating the amplitude of the source to the volume it occupies. By combining equations (2) and (1), the particle velocity vector is eliminated and the inhomogeneous wave equation is derived. It follows from this derivation and from the relations described by Equation (13), that the source term in Equation (6), becomes

$$
\psi(\mathbf{x}, t)=\frac{\partial q(\mathbf{x}, t)}{\partial t}=\frac{\rho_{0}}{V} \frac{d}{d t} Q(t) \delta\left(\mathbf{x}-\mathbf{x}^{\prime}\right)
$$

Physically, the quantity $\psi(\mathbf{x}, t)$ has the dimension of density per unit time squared $\left(\mathrm{Kg} \mathrm{m}^{-3}\right.$ $\mathrm{s}^{-2}$ ), and can be thought of as fluid emergence due to volume acceleration of the source. 
Following Equation (14), it can be seen that a differentiation constraint applies to sources in the wave equation, meaning that volume velocity should be injected as its first time derivative. Observe that the source terms in Equations (1), and (6), are supplemental to the fundamental time-space relationships, that is, if one sets $q(\mathbf{x}, t)=0$ then the homogeneous wave equation is obtained. This indicates that fluid emergence is an additive process, implying a superposition constraint, which numerically means that source nodes should also be evaluated with the FDTD update equations for air.

In order to generate a volume velocity at the source, some mechanical system is required. Such a system would be governed by the laws of motion, and accordingly introduce further modeling constraints. While some mechanical constraints are specific to a chosen transducer, continuous DC flow is something that traditional acoustic transducers generally cannot produce, therefore one would expect that

$$
\int_{-\infty}^{\infty} \psi(\mathbf{x}, t) d t=0
$$

which naturally occurs if the differentiation constraint described in Section III.B is adhered to, and if $q(\mathbf{x}, t)$ is compact in time (i.e. starts at and decays to zero within a finite amount of time). However, if one decides to arbitrarily choose $\psi(\mathbf{x}, t)$, then failure to adhere to this constraint might have detrimental effects, as will be further discussed in Section V.D.

\section{Numerical Constraints}

Finite difference methods are subject to numerical dispersion, which increases as the ratio of the sample rate to the modeled frequency is decreased. This results in waves whose phase velocities are dependent on frequency and on the direction of propagation ${ }^{5}$. When the grid is excited at frequencies prone to substantial dispersion, numerical errors contribute to the resulting response, which not only impair the ability to perform visual analysis, but may also introduce undesired audible artifacts in resulting auralizations ${ }^{29}$.

Accordingly, it is important that high frequencies are removed from the excitation signal to prevent these from contaminating the simulated field, which is here referred to as 
bandwidth constraint. In the case of auralization, where visual inspection of the soundfield is not required, the grid can be excited directly with the program material to be auralized. A more efficient way is to first determine the room's impulse response using a unit impulse, and subsequently obtain the sound signals at the reciever locations via convolution. In such case, bandlimiting can be enforced in the post-processing stage.

When transient phenomena are studied, the grid is excited with a short, impulsive source signal so that possible temporal overlap between reflections is minimized. Such a pulse signal is compact in time and as such can be said to adhere to a time-compactness constraint, which in practice has to be traded-off against the bandwidth constraint. Note that if the excitation signal is not finite in time by definition, it has to be truncated at points selected such that any discontinuity errors are minimal. In addition, the value of all of the signal derivatives up to the truncation order of the scheme would ideally also be zero at simulation onset. However this further requirement has been reported to be prominent only for higher order numerical schemes ${ }^{30}$.

\section{Injection Methods}

Most generally, an excitation signal can be injected via a single or multiple nodes into a grid representing any of the computed acoustic fields. As this paper aims to develop an excitation approach compatible with both Yee and wave-equation schemes, further analysis and formulation will be given from the perspective of a single pressure node excitation.

\section{Hard Sources}

A hard source is the simplest form of grid excitation, in which an acoustic quantity is directly imposed on the source node. This quantity is represented in the discrete domain by the excitation signal $\left.s_{\mathrm{p}}\right|^{n}$, and accordingly, the update equation for a HS node is

$$
\left.p\right|_{\mathbf{i}^{\prime}} ^{n+1}=\left.s_{\mathrm{p}}\right|^{n+1}
$$


where $\mathbf{i}^{\prime}=\left[l^{\prime}, m^{\prime}, i^{\prime}\right]$ denotes the index position of the source. The first thing evident from Equation (16), is that the laws of mass and momentum conservation are not satisfied at the source node, meaning that the HS does not adhere to a superposition constraint. In other words, update equations for air cannot operate over a HS node and any incoming waves get scattered by the source. Accordingly, the node is often loosely thought of as a sound radiating boundary node. This description, however, is not precise, as such an element should adhere to boundary conditions which are not evident in the HS formulation. In addition, one could argue that in a real measurement scenario, a loudspeaker would inevitably be present in the room, and therefore scattering from a HS is not an unrealistic outcome. However, in an FDTD simulation the physical size of the sound radiating node is directly dependent on the spatial sample period, meaning that the scattering effects of the HS are numerically inconsistent.

\section{Soft Sources}

The scattering and low-frequency problems ${ }^{21}$ of hard sources can be overcome by employing soft sources (SS), in which the excitation signal is superimposed on a source node which has already been evaluated by the update equations for the medium. The update equation for a SS node on a pressure grid is therefore

$$
\left.p\right|_{\mathbf{i}^{\prime}} ^{n+1}=\left\{\left.p\right|_{\mathbf{i}^{\prime}} ^{n+1}\right\}+\left.s_{\mathrm{p}}\right|^{n+1}
$$

where $\left\{\left.p\right|_{\mathbf{i}^{\prime}} ^{n+1}\right\}$ represents the result of updating the node with the general update equation for air, that is, Equation (7), or Equations (3), and (4), in the absence of any source terms. Soft sources may have different effects depending on the type of scheme being used. In Yee-type grids, a SS is differentiated due to the staggered nature of the scheme. The update equation for pressure progresses through time in only one half of a step, and the remaining half-step occurs when updating particle velocity, i.e. by evaluating the derivatives of pressure. This inherent differentiation is important as it ensures elimination of a DC component, yet it also severely modifies the spectrum of the outward propagating wave by generating a 
(normally undesired) roll-off in low frequencies.

In wave-equation methods, the SS does not get automatically differentiated, and as such, gives a different result. The outward wave has a spectral content similar to that of $\left.s_{\mathrm{p}}\right|^{n}$, which is a desired feature. Because of this, however, one is not free to arbitrarily choose the excitation signal. More specifically, any existing DC component in the excitation function may cause the ambient pressure in the room to gradually increase. To explain this, let us consider a plane wave of arbitrary amplitude $A$ propagating through the $x$-plane and interacting with a surface of reflection coefficient $\hat{r}$. The total sound pressure along the plane is given by

$$
p(x, t)=A e^{j(\omega t-k x)}+\hat{r} A e^{j(\omega t+k x)}
$$

Accordingly, for $\omega=0$ the sound pressure is uniformly $p=A(1+\hat{r})$ along the plane. Since the SS is being added to existing pressure, then for any $\hat{r}>0$ a pre-existing DC component would constructively superimpose on itself at the source node. This may result in an incremental offset in the response, as will be numerically evaluated in Section V.D. Similar effects have been observed in the field of computational electrodynamics ${ }^{31}$.

Based on digital waveguide analysis, Karjalainen and Erkut ${ }^{14}$ identified the requirement to superimpose, differentiate and scale soft sources in wave-equation FDTD schemes. Their formulation, which shall be further referred to as a Differentiated Soft Source (DSS), is given by

$$
\left.p\right|_{l^{\prime}, m^{\prime}, i^{\prime}} ^{n+1}=\left\{\left.p\right|_{l^{\prime}, m^{\prime}, i^{\prime}} ^{n+1}\right\}+\frac{\rho_{0} c X}{2 A_{\mathrm{w}}}\left(\left.Q\right|^{n+1}-\left.Q\right|^{n-1}\right)
$$

where $A_{\mathrm{w}}$ denotes the cross-sectional area of the waveguide occupied by the source. Note that here the excitation function is explicitly defined as a volume velocity. The formulation adheres to both superposition and differentiation constraint, but being drawn from 1D waveguide theory the scaling factor would only be correct for one dimensional schemes. 


\section{Transparent Sources}

A side effect of all soft sources is that the injected excitation function is modified by the grid's impulse response, which occurs due to the update equations for the medium operating over the source node ${ }^{19}$. It is important to distinguish between the effects of the grid's impulse response which have a minimal effect on the magnitude of the generated soundfield, and the differentiation process which severely modifies the spectrum of the generated wave. Schneider and colleagues ${ }^{19}$ addressed some of these issues by making use of Transparent Sources (TS), which do not scatter incoming waves and do not get modified by the grid's impulse response. The approach requires that the grid's impulse response is measured prior to the simulation stage and is compensated for during simulation. This process can be described mathematically by

$$
\left.p\right|_{\mathbf{i}^{\prime}} ^{n+1}=\left\{\left.p\right|_{\mathbf{i}^{\prime}} ^{n+1}\right\}+\left.s_{\mathrm{p}}\right|^{n+1}-\left.\left.\sum_{\mu=0}^{n} I\right|^{n-\mu+1} s_{\mathrm{p}}\right|^{\mu}
$$

where $\left.I\right|^{n}$ denotes the pre-measured impulse response of the grid, which is obtained by exciting the grid with a unit impulse and capturing the result of updating the source node with the update equation for air $^{19}$. Therefore, TS in a Yee scheme do not only compensate for the grid's impulse response, but also reverse the effects of source differentiation, effectively resulting in a sound field similar to that of a HS but without scattering any incoming waves. In addition, TS suffer from the same low frequency artifacts as $\mathrm{HS}^{21}$. It should also be noted that the grid's impulse response must be obtained in the absence of any scattering objects, which for long simulation times entails modeling a large domain and thus introduces an additional computational burden. In sum, it can be said that TS do not adhere to any scaling constraints and, due to the grid compensation process, nor to the differentiation constraint. 
(a)

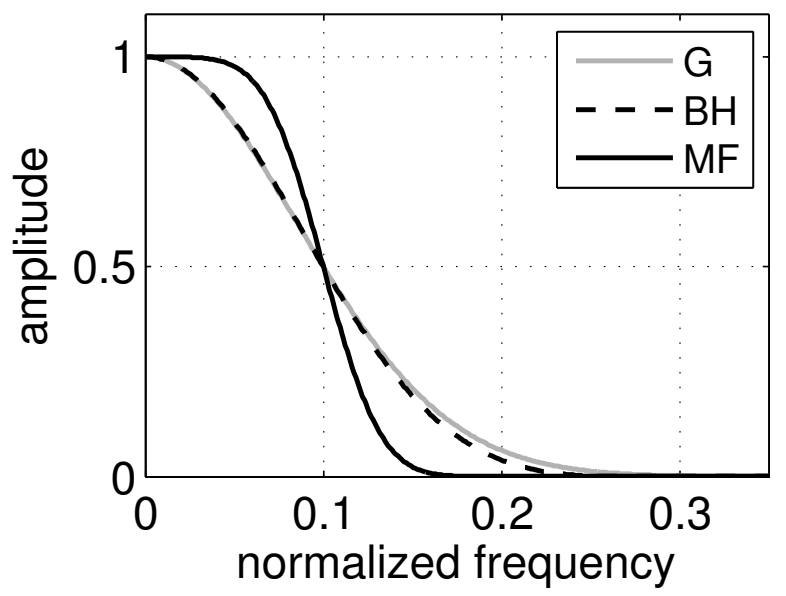

(b)

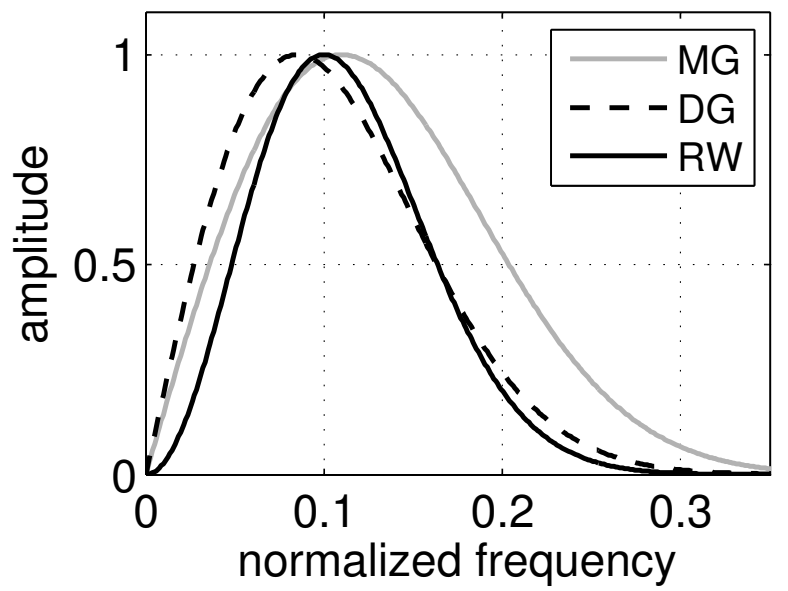

FIG. 1. Pulse spectra. (a) Gaussian (G), Blackman-Harris (BH), and Maximally Flat (MF) FIR pulse. (b) Differentiated Gaussian (DG), Sine-Modulated Gaussian (MG), and Ricker Wavelet (RW). The modulation frequency for the MG pulse and the peak frequency of the RW pulse were chosen equal to the cutoff frequeny $f_{c}=0.1 f_{s}$.

\section{E. Pulse Shaping}

The grid has to be excited with a pulse signal that adheres to the aforementioned bandwidth and the time-compactness constraints, and is usually defined in terms of a $-6 \mathrm{~dB}$ cutoff frequency $\left(f_{c}\right)$ and the number of samples $(M)$. Two widely employed pulse signals in FDTD modeling are the Gaussian pulse and the Blackman-Harris window ${ }^{12}$. Figure 1(a) shows the respective amplitude spectra for $f_{c}=0.1 f_{s}$ and $M=79$. The Gaussian pulse signal has to be truncated with care in order to avoid the introduction of spectral ripples. The Blackman-Harris pulse has inherent stopband ripples, and any detrimental effects may become particularly evident when lower cutoff frequencies are required ${ }^{12}$.

Differentiated versions of these pulse signals are sometimes used in order to avoid DC excitation $^{11,12}$. A special case is the Ricker wavelet ${ }^{32}$, which is a normalized second-derivative of a Gaussian function, and has several documented uses in acoustics FDTD ${ }^{20,33,34}$. In the light of the discussion in Section III.B, it can be said that the differentiation constraint is inherently met when using such pulses. Similarly, sine-modulated pulses ${ }^{12,21}$ have no DC 
component and may be considered as differentiated versions of pulse signals of finite power and length, thus also meeting the differentiation constraint. Figure 1(b) shows a spectral comparison between a Ricker wavelet, a differentiated Gaussian and a sine-modulated Gaussian.

It is worthwhile noting that the differentiation in Equation (14), stems from the governing equations, which are discretized in the numerical formulation. It is therefore more consistent with the FDTD model to incorporate the source differentiation in the same discretized fashion, rather than performing an analytic differentiation on the initial pulse signal. As explained in Section IV, this leads to the use of an "injection filter" for wave equation FDTD grids.

The main remaining assessment criterion is the extent to which the pulse spectrum is flat and rippleless in its passband and stopband. As such, a good alternative to the standard Gaussian and Blackman-Harris pulses can be found in the digital signal processing literature on maximally flat (MF) FIR lowpass filter design. In the original formulation ${ }^{35}$, the MF FIR tap coefficients were computed by applying an inverse discrete Fourier transform to polynomial expressions evaluated in the frequency domain. More recently, Khan and Ohba ${ }^{36}$ derived explicit formulae, from which an MF pulse can be defined for $-(2 N-1) \leq n \leq$ $(2 N-1)$ as

$$
\begin{aligned}
& \left.s_{\mathrm{p}}\right|^{0}=\omega_{c} T \\
& \left.s_{\mathrm{p}}\right|^{n}=\frac{(2 N-1) ! !^{2} \sin \left(n \omega_{c} T\right)}{\hat{b} n(2 N+n-1) ! !(2 N-n-1) ! !}
\end{aligned}
$$

where the coefficient $\hat{b}$ equals 2 for odd $n$ and $\pi$ for even $n, \omega_{c}=2 \pi f_{c}$ is the angular cutoff frequency and $M=4 N-1$. As seen in Figure 1(a), the MF pulse spectrum is flatter within the pass band than the standard pulse signals, and also has a steeper roll-off. Together with the absence of stopband ripples this makes the MF FIR pulse particularly suited to FDTD field visualization and auralization. 


\section{UNIFIED SOURCE MODELING USING CASCADED FILTERS}

In order to gain a stronger sense of overview over the design process, it is useful to represent the source model in terms of its associated signal processing path. As such, the process of injecting a source signal can be generalized in parametric fashion by considering it as a system of three cascaded digital filters whose input is a Kronecker Delta, as shown in Figure 2. The delta function is first passed through a pulse shaping filter of transfer function $H_{\mathrm{p}}(z)$, which ensures that the system is driven using a signal adhering to the

aforementioned numerical constraints. The output of this filter is the excitation signal $\left.s_{\mathrm{p}}\right|^{n}$, which then drives a mechanical filter of transfer function $H_{\mathrm{m}}(z)$, the function of which is to meet some of the transduction constraints. In principle, removal of a DC component can be accomplished by means of a simple DC-blocker ${ }^{37}$, but - as shown in Section IV.A - a more systematic approach is to simulate the mechanics of a simple transducer.

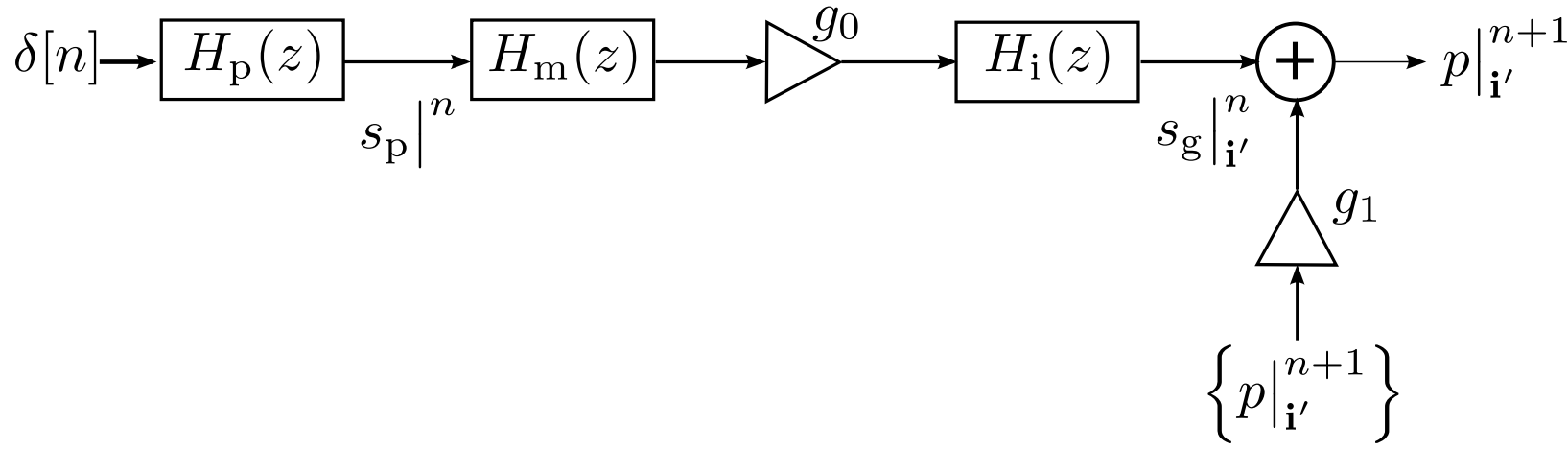

FIG. 2. Unified representation of source models. $H_{\mathrm{p}}(z)$ pulse-shaping filter, $H_{\mathrm{m}}(z)$ mechanical filter, $H_{\mathrm{i}}(z)$ injection filter, $\left.s_{\mathrm{p}}\right|^{n}$ excitation signal, $\left.s_{\mathrm{g}}\right|_{\mathbf{i}^{\prime}} ^{n}$ final grid signal to be injected.

The remaining transduction constraints are then met by employing an injection filter, $H_{\mathrm{i}}(z)$, and its corresponding gain coefficients $g_{0}$ and $g_{1}$. This represents the final stage in transforming the excitation signal $\left.s_{\mathrm{p}}\right|^{n}$ into the source function $\left.s_{\mathrm{g}}\right|_{\mathbf{i}^{\prime}} ^{n}$. The purpose of the coefficient $g_{0}$ is to account for the scaling constraints. The signal is then routed through an injection filter which acts either as a differentiator or, for a transparent source, as a cancellation mechanism. Lastly, the gain function $g_{1}$ controls the superposition constraint, 
and may take on the values 0 or 1 depending on whether the source function is imposed or superimposed on the grid. While the two filters, $H_{\mathrm{i}}(z)$ and $H_{\mathrm{m}}(z)$, are associated with the same physical system, they are here described separately in order to allow an efficient generalization of FDTD source models.

\section{A. Physically Constrained Source (PCS) Model}

The unified source representation directly facilitates the design of source models that adhere to the aforementioned constraints. In this section, such a model is derived starting from a pulsating sphere of (small) radius $a_{0}$ whose surface velocity $\nu(t)$, in vacuum, is governed by

$$
M \frac{\partial \nu(t)}{\partial t}=-R \nu(t)-K \int \nu(t) d t+F(t)
$$

where $M, R$, and $K$ are respectively, the mass, damping and elasticity constants characterizing the mechanical system, and $F(t)$ is the mechanical force driving the sphere pulsation (not to be confused with acoustic force, which has been neglected in this formulation). With air surrounding the sphere, the mechanical impedance of the system is $Z(\omega)=Z_{\mathrm{V}}(\omega)+Z_{\mathrm{a}}(\omega)$ where

$$
Z_{\mathrm{V}}(\omega)=M j \omega+R+K /(j \omega)
$$

is the impedance of the system in vacuum and

$$
Z_{\mathrm{a}}(\omega)=\rho_{0} A a_{0}\left(j \omega+\left(a_{0} / c\right) \omega^{2}\right)
$$

is the mechanical impedance of the surrounding $\operatorname{air}^{38}$, approximated for $k a_{0} \ll 1$. However, the latter term may be omitted since $a_{0}$ is very small, meaning that $\left|Z_{\mathrm{V}}(\omega)\right| \gg\left|Z_{\mathrm{a}}(\omega)\right|$ in all practical cases. Hence the system may be characterized by the transfer function

$$
H_{\mathrm{m}}(s)=\frac{s}{M s^{2}+R s+K}
$$

which has the dimension of mechanical admittance. In the time domain, the impulse response of the system is given by

$$
h_{\mathrm{m}}(t)=\left[\cos \left(\omega_{\mathrm{r}} t\right)-\frac{\alpha}{\omega_{\mathrm{r}}} \sin \left(\omega_{\mathrm{r}} t\right)\right] M e^{-\alpha t}
$$


where $\alpha=R /(2 M)$ is the damping factor, $\omega_{0}=\sqrt{K / M}$ is the system's undamped resonant frequency and $\omega_{\mathrm{r}}=\sqrt{\omega_{0}^{2}-\alpha^{2}}$. At the source, the sphere's surface velocity equals the particle velocity of air, which can be mathematically expressed as convolution between the driving force and the system's impulse response, $\nu(t)=F(t) * h_{\mathrm{m}}(t)$. The pulsation of the sphere causes fluid to be pushed into and extracted from the region bordering the source sphere surface, which is characterized by a volume velocity,

$$
Q(t)=\nu(t) A_{\mathrm{s}}
$$

having the dimension of volume per unit time, where $A_{\mathrm{s}}=4 \pi a_{0}^{2}$ is the surface area of the sphere.

In the numerical domain, the transfer function of the PCS mechanical filter, $H_{\mathrm{m}}(z)$, can be formulated by applying a bilinear transform to $H_{\mathrm{m}}(s)$. This choice is mainly because, unlike other discretization methods, the bilinear transform does not place any stability limits on the values of $M, R$ and $K$, thus allowing them to be freely chosen. Taking the bilinear transform of Equation (25), the following digital filter is obtained:

$$
H_{\mathrm{m}}(z)=\frac{b_{0}+b_{2} z^{-2}}{1+a_{1} z^{-1}+a_{2} z^{-2}}
$$

with the coefficients given by

$$
\begin{array}{ll}
b_{0}=\frac{\beta}{M \beta^{2}+R \beta+K} & b_{2}=-\frac{\beta}{M \beta^{2}+R \beta+K} \\
a_{1}=\frac{2\left(K-M \beta^{2}\right)}{M \beta^{2}+R \beta+K} & a_{2}=1-\frac{2 R \beta}{M \beta^{2}+R \beta+K}
\end{array}
$$

where $\beta$ is the bilinear operator, which for a pre-warped $\omega_{0}$ is given by

$$
\beta=\frac{\omega_{0}}{\tan \left(\omega_{0} T / 2\right)}
$$

In the PCS method one considers the quantity represented by the excitation signal $\left.s_{\mathrm{p}}\right|^{n}$ to describe the mechanical force driving the sphere, that is, the discrete time equivalent of $F(t)$. Passing this signal through $H_{\mathrm{m}}(z)$ yields the sphere's surface velocity $\left.\nu\right|^{n}$, which is then used in the final injection network. 
In this formulation, the pulsating sphere is thought of as an external entity, unidirectionally coupled to the grid but not embedded into it, whose sole purpose is to generate a prescribed volume velocity. When this quantity is applied to a single grid node, the spatial period and nodal density of the rectilinear grid dictate that fluid emerges within a finite volume of $V=X^{3}$. Accordingly, by discretizing Equation (13), a numerical equivalent of $q(\mathbf{x}, t)$ is given by

$$
\left.q\right|_{\mathbf{i}^{\prime}} ^{n}=\left.\frac{\rho_{0} A_{\mathrm{s}}}{X^{3}} \nu\right|^{n} \delta\left[\mathbf{i}-\mathbf{i}^{\prime}\right]
$$

To derive the PCS injection filter and its corresponding coefficients $g_{0}$ and $g_{1}$, one needs to consider the type of scheme being used. Taking into account the additional scaling factors for the source term in Equation (3), the coefficient $g_{0}$ for a Yee-type scheme is given by

$$
g_{0}=\frac{z_{0} \lambda A_{\mathrm{s}}}{X^{2}}
$$

Since in a Yee-scheme source differentiation is inherent in the update equations, the transfer function of the injection filter's is $H_{\mathrm{i}}(z)=1$. Considering the superposition constraint, $g_{1}$ is set to unity in order to allow the update equation for air to operate over the source node. Accordingly, the final update equation for a Yee-type source node becomes

$$
\begin{aligned}
\left.p\right|_{\mathbf{i}^{\prime}} ^{n+1} & =\left\{\left.p\right|_{\mathbf{i}^{\prime}} ^{n+1}\right\}+\left.g_{0} \nu\right|_{\mathbf{i}^{\prime}} ^{n+1} \\
& =\left\{\left.p\right|_{\mathbf{i}^{\prime}} ^{n+1}\right\}+\left.\left(c^{2} T\right) q\right|_{\mathbf{i}^{\prime}} ^{n+1}
\end{aligned}
$$

which is equivalent to the formulation proposed by Matheson ${ }^{39}$. To develop the injection filter for the wave equation method, the physical definition of $\psi(\mathbf{x}, t)$ is followed. In the numerical domain, the differentiation constraint described by Equation (14), is adhered to by employing central finite differences approximating the time derivative of $q(\mathbf{x}, t)$. Accordingly, the transfer function of the injection filter for the wave equation is

$$
H_{\mathrm{i}}(z)=\frac{1}{2 T}\left(z-z^{-1}\right)
$$

Considering the scaling constraints drawn from the formulation of $\left.q\right|_{\mathbf{i}^{\prime}} ^{n}$, the coefficient $g_{0}$ for a wave-equation source is given by

$$
g_{0}=\frac{\lambda^{2} \rho_{0} A_{\mathrm{s}}}{X}
$$


Adhering to the superposition constraint, $g_{1}$ is set to unity, and the final update equation for a wave-equation source node becomes

$$
\begin{aligned}
\left.p\right|_{\mathbf{i}^{\prime}} ^{n+1} & =\left\{\left.p\right|_{\mathbf{i}^{\prime}} ^{n+1}\right\}+\frac{g_{0}}{2 T}\left(\left.\nu\right|_{\mathbf{i}^{\prime}} ^{n+1}-\left.\nu\right|_{\mathbf{i}^{\prime}} ^{n-1}\right) \\
& =\left\{\left.p\right|_{\mathbf{i}^{\prime}} ^{n+1}\right\}+\frac{c^{2} T}{2}\left(\left.q\right|_{\mathbf{i}^{\prime}} ^{n+1}-\left.q\right|_{\mathbf{i}^{\prime}} ^{n-1}\right)
\end{aligned}
$$

\section{B. Generalizing Source Models}

The signal processing chain described in this section can be used to generalize the process of modeling sources for FDTD simulation, where all existing source models, as well as the PCS, can be seen as special cases of the cascaded-filters method. To summarize this, Table I shows the different transfer functions and coefficients which may be used in the filter network in order to model different sources. For hard and soft sources the grid source function simply equals the excitation signal at the source position, that is $\left.s_{\mathrm{g}}\right|_{\mathbf{i}^{\prime}} ^{n}=\left.s_{\mathrm{p}}\right|^{n}$ with the only difference being the value of $g_{1}$ which controls the superposition constraint. Within our formulation, in a Yee-type scheme the dimension of a hard source is pressure and the dimension of a soft source is velocity (due to the inherent differentiation), whereas in wave equation schemes both sources have the dimension of pressure. Differentiated soft sources calculate the signal's time derivative in the injection filter and therefore the injected quantity is volume velocity, however, their associated scaling coefficient $g_{0}$ is appropriate for 1D grids. Transparent sources feature a processing chain similar to that of soft sources, with the injection filter designed to compensate for the grid IR and, in Yee-schemes also reverse the effects of inherent differentiation. For the PCS method, the dimension of $\left.s_{\mathrm{p}}\right|^{n}$ is mechanical force and, after the complete signal processing chain, the source function represents source density (in Yee

methods), i.e. $\left.q\right|_{\mathbf{i}^{\prime}} ^{n}=\left.s_{\mathrm{g}}\right|_{\mathbf{i}^{\prime}} ^{n}$, or its first time derivative (in wave equation methods), i.e. $\left.\psi\right|_{\mathbf{i}^{\prime}} ^{n}=\left.s_{\mathrm{g}}\right|_{\mathbf{i}^{\prime}} ^{n}$.

Readers who wish to make practical use of the unified source representation described in this section may download a dedicated Matlab function library, the Source Modeling Toolbox, which has been made available online ${ }^{40}$. 


\section{RESULTS AND DISCUSSION}

\section{A. Prescribed Pressure}

To exemplify how the PCS can be designed to achieve a prescribed pressure field, a receiver was placed at the center of a $6 \times 6 \times 6 \mathrm{~m}$ domain, which was solved using the standard rectilinear scheme $(a=0$ and $b=0)$ at a sample rate of $16 \mathrm{kHz}$. A PCS was placed at a radial distance of $r=1.5 \mathrm{~m}$ and an azimuth of $45^{\circ}$ on the same plane as the receiver. The simulation was executed long enough for the entire signal to propagate from the source to the receiver but without introducing any reflections from the boundaries. The excitation signal was designed with the impulse response of a MF FIR $\left(M=16\right.$ and $\left.f_{c}=0.075 f_{s}\right)$, which corresponds to the $2 \%$ dispersion criterion for the standard rectilinear scheme ${ }^{5}$. The magnitude of excitation was chosen such that the peak amplitude of the filter's output is normalized to a driving force of $250 \mu \mathrm{N}$.

The mechanical filter of the PCS is characterized by the system resonance $\omega_{0}$ and quality factor $\mathcal{Q}$. In an optimal transducer design process, the designer would specify the desired values for these parameters and the remaining electro-mechanical quantities would be engineered accordingly. In this experiment, the radius of the pulsating sphere was arbitrarily chosen to be $a_{0}=5 \mathrm{~cm}$, and its mechanical constants corresponded to values of $M=25 \mathrm{~g}$, $f_{0}=100 \mathrm{~Hz}$ and $\mathcal{Q}=0.7$. It is worthwhile noting that a transducer of such small surface area would, in reality, produce a poor volume velocity at low frequencies. However, while the numerical model is governed by physical laws, it is not bound by real world engineering constraints, and as such, it is possible to design a small sphere of such low resonance. Accordingly, the remaining damping and stiffness coefficients are calculated from $R=\omega_{0} M / \mathcal{Q}$ and $K=M \omega_{0}^{2}$, respectively. As reference, a closed-form solution for Equation (6) in free field is used. With a point-source approximation, the sound pressure at the distance $r=\left\|\mathbf{x}-\mathbf{x}^{\prime}\right\|$ is given $b^{28}$

$$
p(r, t)=\frac{\rho_{0}}{4 \pi r} \frac{d}{d t} Q\left(t-\frac{r}{c}\right)
$$

Numerical results were obtained using both the wave equation method and the Yee-type 
method, and a reference response was calculated by passing the PCS volume velocity through Equation (37). As shown in Figure 3(a), when using the PCS model, both methods are in agreement with the closed form solution.

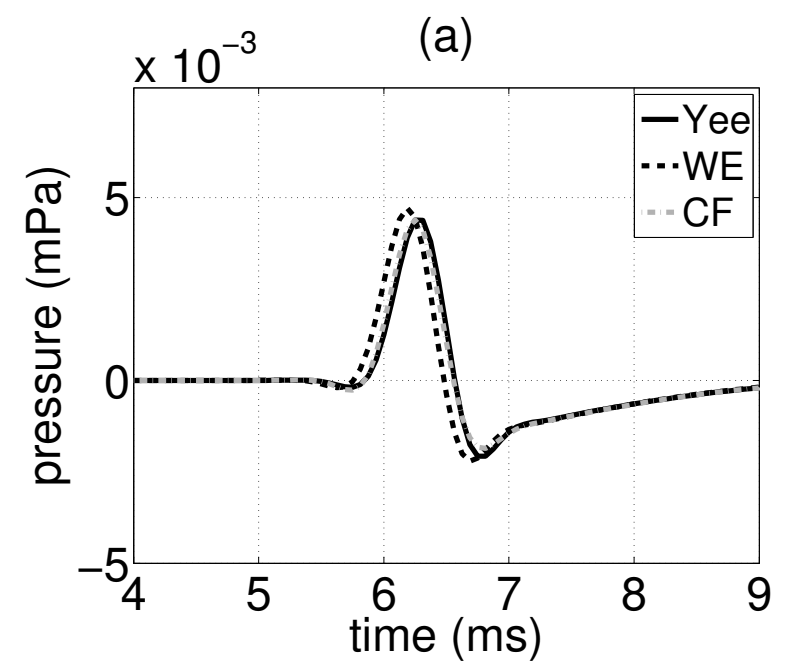

(b)

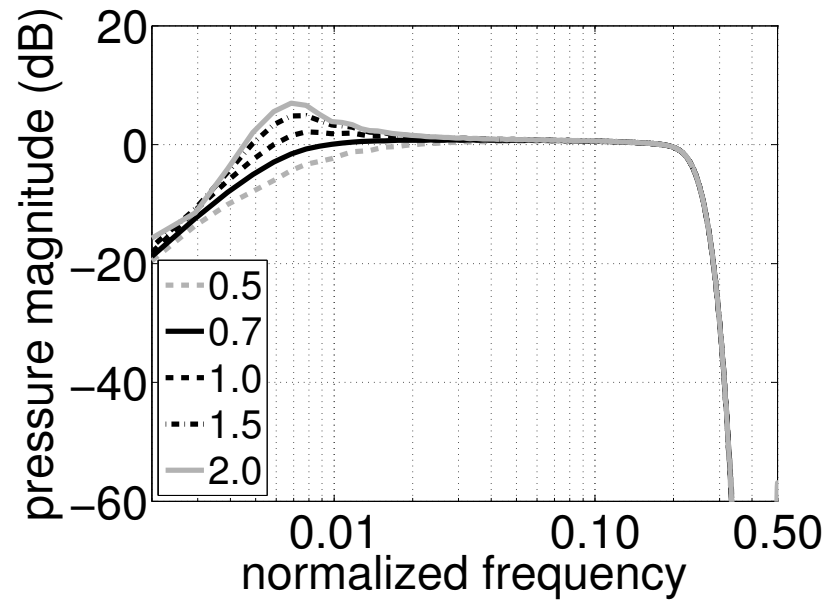

FIG. 3. Sound pressure at the receiving position of a domain excited using the PCS method. (a) Time domain comparison: Yee and Wave equation (WE) methods plotted against the closed-form solution (CF). (b) Frequency spectra: wave equation method solved with different values of $\mathcal{Q}$.

\section{B. Frequency Response Comparison}

To study the pressure spectrum resulting from a PCS excitation, the same experiment was conducted using an interpolated wideband scheme $(a=1 / 4$ and $b=1 / 16)$, allowing for the high cutoff frequency to be increased to $0.25 f_{s}$. The PCS resonance was kept at $f_{0}=100 \mathrm{~Hz}$, which corresponds to $0.0063 f_{s}$. This simulation was repeated for different values of $\mathcal{Q}$ ranging from 0.5 to 2.0. As seen in Figure 3(b), the PCS model facilitates a means to design sources having a flat bandwidth between the system's resonance and the cutoff frequency of the pulse-shaping filter. As expected from a second order linear system, adjusting $\mathcal{Q}$ controls the trade-off between the steepness of the low-frequency transition band and the magnitude of resonance. 
For comparison of with other source models, three simulations were executed using an interpolated-wideband scheme, with a HS (also representative of the frequency response of a TS and a wave-equation SS), a DSS (also representative of a Yee-type SS) and a PCS. All simulations used a MF FIR pulse with $f_{c}=0.25 f_{s}$, and the PCS was designed with a low resonance at $f_{0}=0.167 f_{s}$ and $\mathcal{Q}=0.7$. For visual clarity, simulation outputs were normalized such that the peak value of each resulting impulse response is unity. As seen in Figure 4, the SS suffers from a severe roll off at low frequencies, which is to be expected due to differentiation (be it inherited in the source formulation or in the grid update equations in the case of a Yee method). Given that in the standard SS formulation, no mechanical or pulse shaping filter is explicitly defined, either the flatness requirement is not met (if the signal is differentiated) or solution growth is not prevented (if it is undifferentiated). In the PCS model, the mass reactance of the sphere acts as an integrator which, in a physical manner, counters the effects of differentiation. Below its resonant frequency, the system is stiffness controlled, and as such, naturally acts as a DC-blocking filter. The result is a source having a near-flat pressure spectrum whose physical properties can be freely chosen by adjusting $\mathcal{Q}$ and $\omega_{0}$. In comparison to a HS, the spectrum of the PCS is flat above $f_{0}$ but not down to DC; however, such a low-frequency response is essential for the exclusion of a DC component.

\section{Numerical Consistency}

When simulating a physical system, changing numerical parameters should only affect the accuracy of the model. Accordingly, changing the sample rate of an FDTD model should not affect the magnitude of the generated sound field, a notion which is related to the scaling constraint discussed in Section III. To test this, the wave equation FDTD method was used with three sources, namely HS, DSS and PCS. Transparent sources and undifferentiated soft sources have the same scaling coefficients as HS, thus as far as the magnitude of the soundfield is concerned, results can be appropriately deduced from the HS example. The simulation 


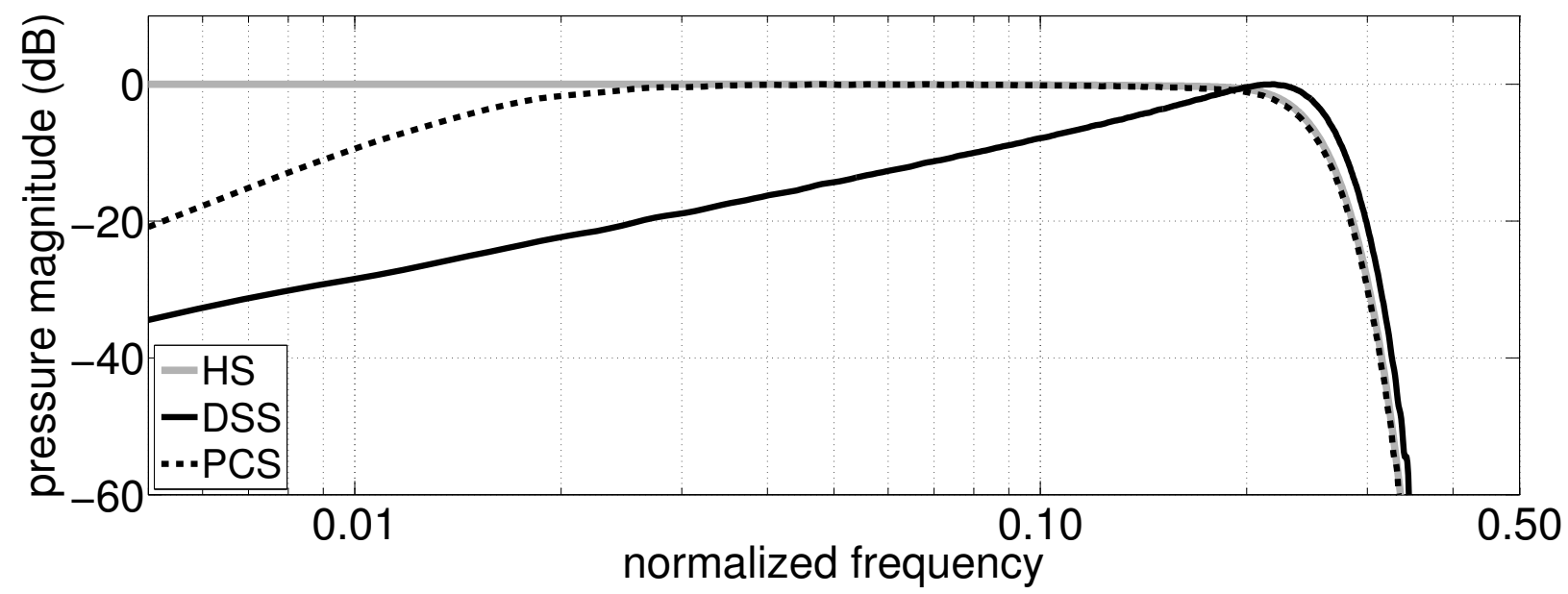

FIG. 4. Calculated frequency response for three different source models, HS - hard source (response similar to TS), DSS - differentiated soft source (response similar to SS in Yee methods), PCS - physically constrained source. Excitation signals are MF FIR pulses of $N=16$ and $f_{c}=0.25 f_{s}$. PCS resonance is at $f_{0}=0.167 f_{s}$.

was repeated for three sample rates, namely $8 \mathrm{kHz}(X=74.37 \mathrm{~mm}), 12 \mathrm{kHz}(X=49.58 \mathrm{~mm})$ and $18 \mathrm{kHz}(X=33.05 \mathrm{~mm})$. An MF-FIR pulse-shaping filter with $M=16$ and $f_{c}=600 \mathrm{~Hz}$ was used in all simulations (regardless of the sample rate), thus ensuring that anomalies do not occur due to differences in the excitation signals.

It can be seen in Figure 5 that the PCS is the only source model which results in a response whose magnitude is independent of sample rate. Nevertheless, in a one-dimensional problem, one would expect similar consistency for the case of a differentiated soft source, when it is appropriately scaled as described by Karjalainen and Erkut ${ }^{14}$.

\section{DC and Low Frequency Artifacts}

The theoretical analysis in Section III.D indicates that when soft sources in wave equation schemes include a DC component, a growing solution could occur. The concern arises when one uses an arbitrary SS, such as described by Equation (19), where the source function directly equals the excitation signal, and as such, may contain energy around DC. To test 
(a)

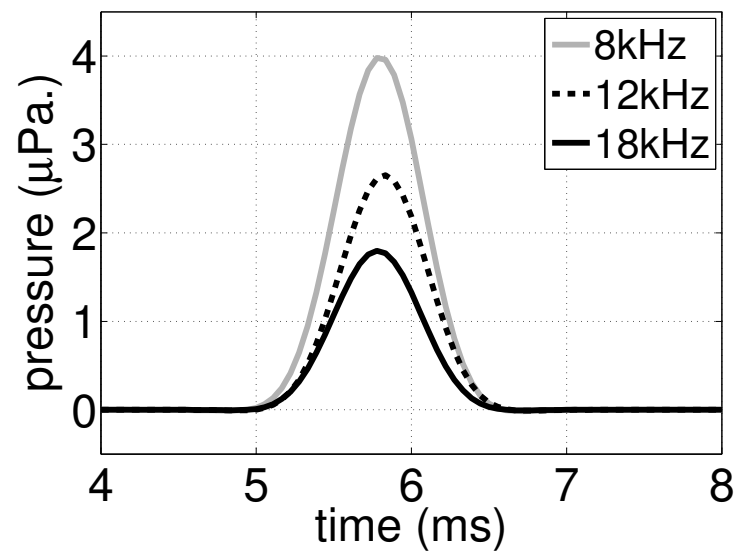

(b)

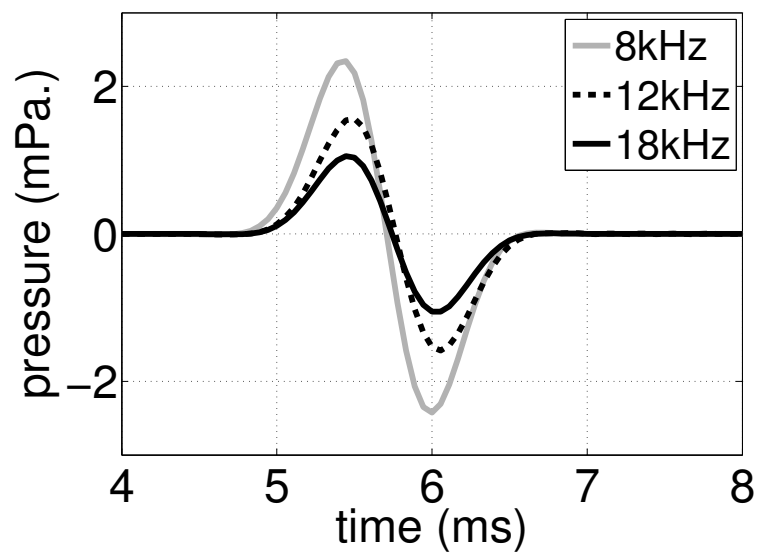

(c)

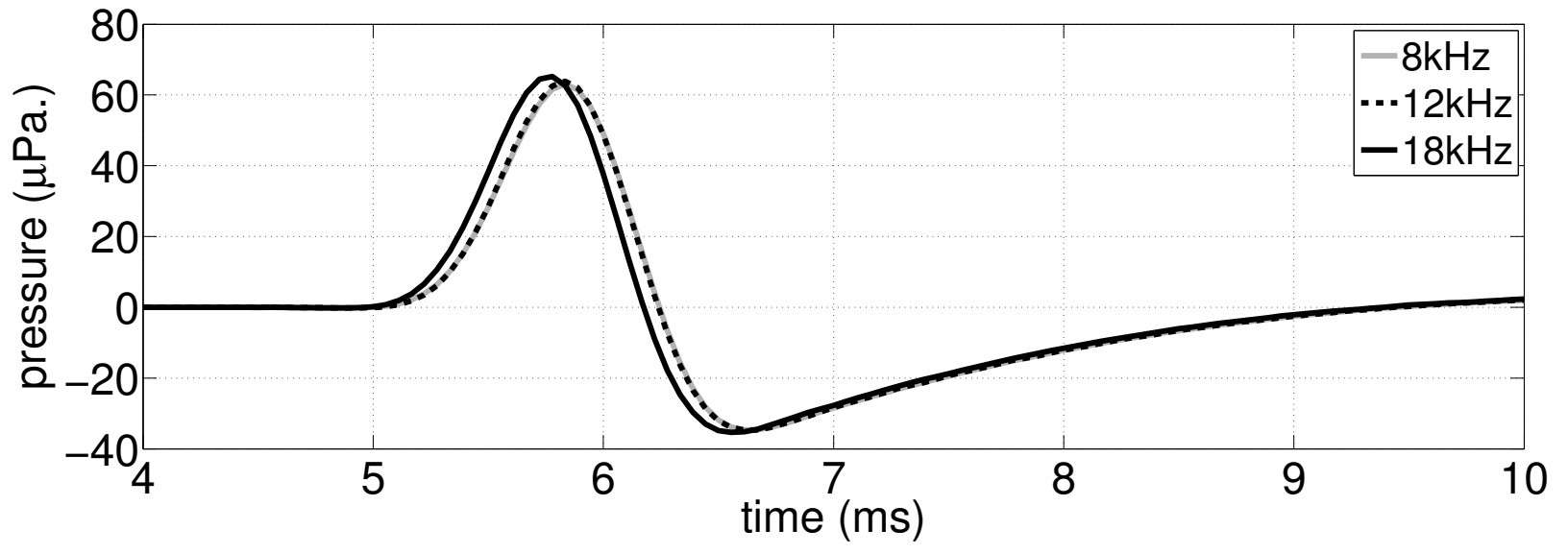

FIG. 5. Pressure at the receiving position of a grid excited by (a) hard-source, (b) differentiated soft-source and (c) physically-constrained source, at three different sample rates.

this, let us consider an arbitrary SS and a PCS, both of which are designed using a Gaussian pulse shaping filter. This pulse is unipolar and hence features a strong DC component. A receiver was placed at the center of a $216 \mathrm{~m}^{3}$ room at a distance of $0.5 \mathrm{~m}$ from the source. The room was designed with uniform frequency independent boundaries, corresponding to a normal-incidence reflection coefficient of $\hat{r}=0.997$. Results from these simulations are displayed in Figure 6(a). For visual clarity, responses are normalized such that the direct component in the resulting responses equal 1Pa. It is evident that the PCS response remains around the horizontal axis over time, whereas the soft source solution is linearly growing. This growth is attributed to the accumulation of DC in the soundfield, and is unrelated to 
stability issues which normally cause an exponential growth.

(a)

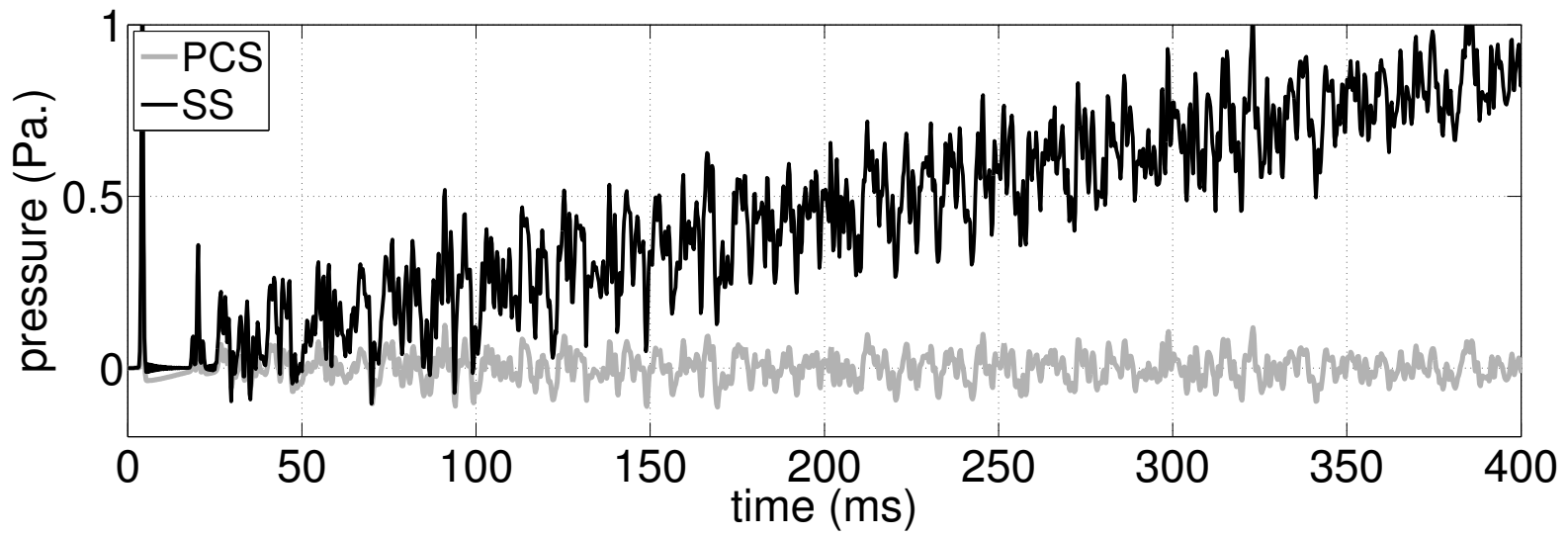

(b)

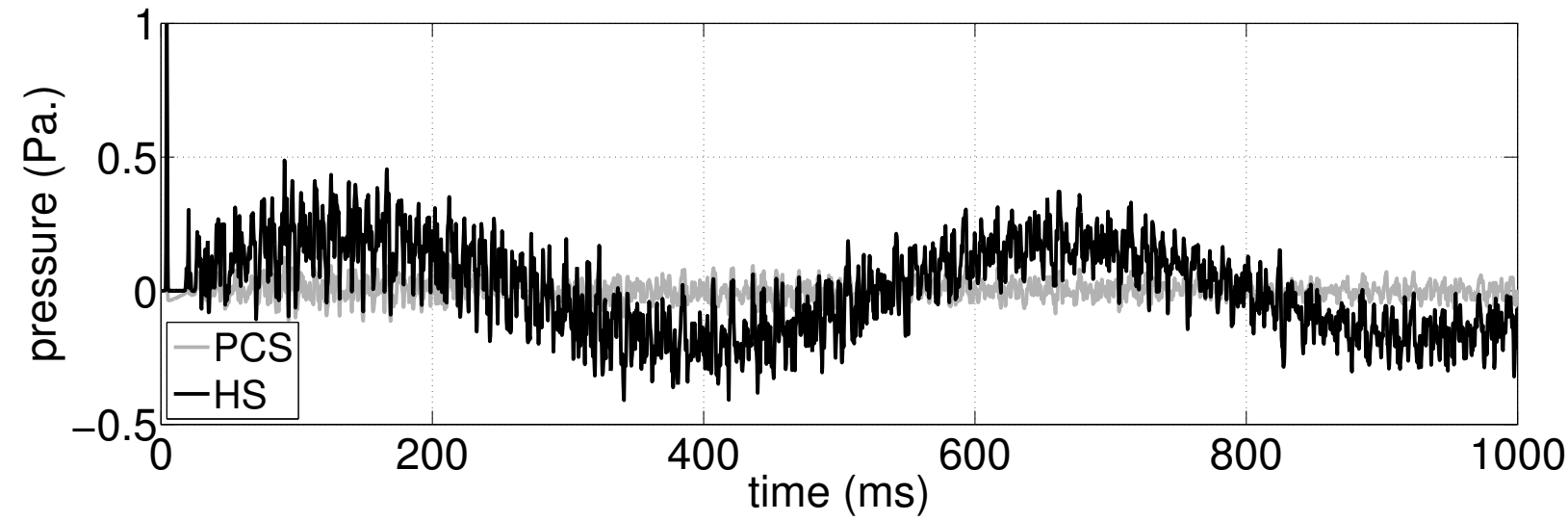

FIG. 6. Sound pressure at the receiving position for a grid excited by a physically constrained source (PCS) compared to (a) SS - undifferentiated soft source and, (b) HS - hard source. All source models employ a Gaussian pulse shaping filter $\left(\sigma=3 \frac{1}{3} \cdot 10^{-4}\right)$. Results are normalized for visual clarity.

Such a growth is also sensible from a physical perspective as a DC component in $\left.s_{\mathrm{g}}\right|^{n}$ indicates that $q(t)$ is not of finite length, meaning that the equivalent excitation signal does not adhere to a time-compactness constraint. To explain this, it is useful to discuss the physical meaning of using the Gaussian as a source function in an undifferentiated SS model. Since such a source does not adhere to the differentiation constraint nor to any other mechanical constraints, then the excitation signal and source function are a direct numerical 
representation of $\psi(\mathbf{x}, t)$ :

$$
\left.s_{\mathrm{g}}\right|^{n}=\left.\left.s_{\mathrm{p}}\right|^{n} \equiv \psi(\mathbf{x}, t)\right|_{t=n T}
$$

Since $\psi(\mathbf{x}, t)$ is defined as the first time derivative of $q(\mathbf{x}, t)$, then following Equation (14), the rate of fluid emergence due to the soft source is obtained by taking the integral of a Gaussian function, which yields

$$
\begin{aligned}
q(\mathbf{x}, t) & =\int \psi(\mathbf{x}, t) d t=\int A_{p} \exp \left(\frac{-\left[t-t_{0}\right]^{2}}{2 \sigma^{2}}\right) d t \\
& =\sqrt{\frac{\pi}{2}} A_{p} \sigma \mathrm{ERF}\left(\frac{t-t_{0}}{\sqrt{2} \sigma}\right)
\end{aligned}
$$

where $\operatorname{ERF}(\cdot)$ is the Gauss error function, $\sigma$ is the pulse variance, $A_{p}$ is the amplitude of the pulse and $t_{0}$ denotes an initial time shift. Figure 7 depicts $\psi(t)$ and $q(t)$, for such an undifferentiated soft source and for a physically constrained source.

(a)

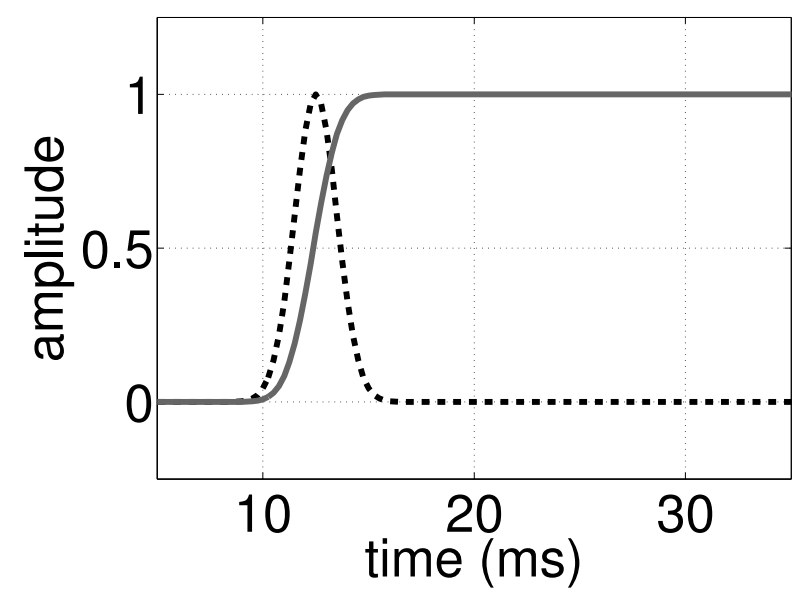

(b)

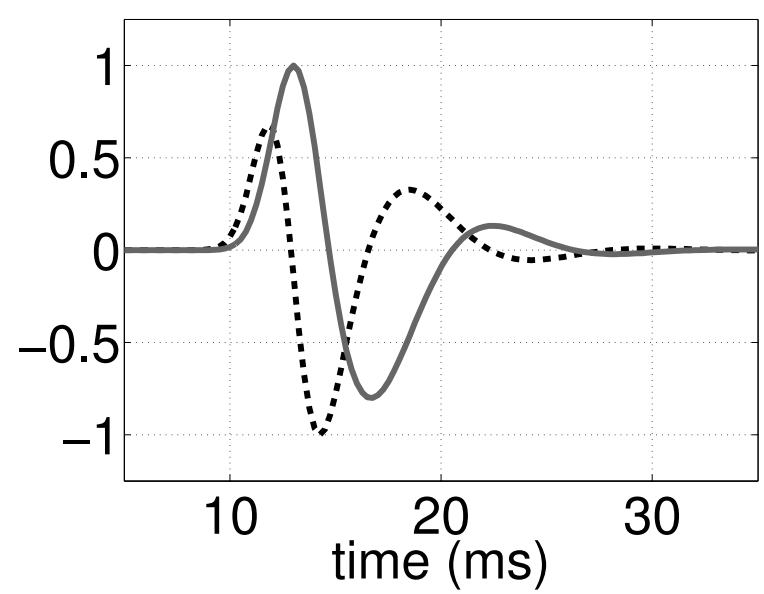

FIG. 7. Source function (dashed lines), $\psi(t)$, and rate of fluid emergence (solid lines), $q(t)$ at the source node, for (a) undifferentiated soft source and (b) physically constrained source, both excited using a Gaussian pulse. Results have been normalized to $\pm 1 \mathrm{~Pa}$. and $\pm 1 \mathrm{kgm}^{-3} \mathrm{~s}^{-1}$ for visual clarity.

When the PCS mechanical filter is damped (i.e. $\alpha>0$ ) and driven by an appropriately time-limited force, then both $q(t)$ and $\psi(t)$ start at and decay to zero, indicating a finite 
source. However, this is not the case for the arbitrary SS. The fact that the grid signal represented by $\psi(t)$ is time-limited can be misleading as, in physical terms, it only means that the source generating mechanism does not accelerate before or after the excitation period. This does not mean that the source is not active. In fact, it can be seen for the SS that when $\psi(t)$ decays, $q(t)$ rises and stays at a constant value through the remaining simulation period, which indicates that even when $\psi(t)$ is time limited, the source mechanism may still generate volume velocity. As one would expect, $q(t)$ remains at a constant positive value which is equivalent to the generation of DC flow, meaning that the soundfield continuously gets pressurized by the source.

For the case of a HS injection, solution growth is not expected even if the excitation signal contains a DC component. This is because hard sources do not adhere to the superposition constraint, and as such, the existing pressure at the source node gets replaced by (rather than added to) the source function. As was identified by Jeong and Lam ${ }^{21}$, this prevents air particles at the source position from being able to perform rarefaction, which leads to a spurious low frequency component in the resulting response. Figure 6(b) compares the results of exciting the grid with a PCS and HS, both of which are based on a Gaussian excitation signal. It can be seen that while the HS solution does not display growth, it does contain a spurious low frequency component (with a period of 582ms).

\section{E. Time Limiting}

Based on the assumption that excitation signals are relatively compact in time, it was further suggested by Jeong and $\mathrm{Lam}^{21}$ that the HS scattering and low frequency artifacts can be overcome by using sine-modulated pulses together with time-limiting the source injection

process. To accomplish this, the source node is updated with a HS formulation until the associated excitation signal has decayed to zero, after which the regular update equations for the medium are used. This workaround may appear useful for generating a soundfield similar to that of a transparent source, however it bears a couple of complications. Firstly, 
even if the excitation signal has decayed to zero, one cannot generally assume that the nodes surrounding the source are also null (although if the excitation signal is short and the source is sufficiently distant from a boundary, they might be). Additionally, it was shown in Figure 7 that in wave equation methods it is possible that even when the source function has decayed, the source is still physically active. Since the update equations for the medium involve temporal as well as spatial differentiation, any sudden change in the equations for the source node might introduce errors arising from the associated discontinuities.

\section{CONCLUDING REMARKS}

A coherent approach to modeling sources in acoustic FDTD simulation has been made possible by representing the signal injection path with a chain of digital filters, and deriving the associated parameters from the physics and the numerics of the problem. The results presented in Section V show that a simple numerical monopole source can be formulated which is consistent with its continuous-domain counterpart, does not scatter wave energy, and effects a free-field pressure wave that is spectrally flat between specified cut-off frequencies. As such, the proposed physically-constrained source model offers an improved approach for meeting the aims and constraints inherent to FDTD excitation.

One principal limitation remains, in that the design of the source signal cannot escape the Gabor limit, meaning that there is inevitably some limit on the simultaneous time-frequency resolution one may achieve. Within this fundamental restriction, the proposed method offers some design freedom through control of the resonance frequency and quality factor of the modeled pulsating sphere, both of which are intuitive design parameters from a physical as well as a spectral analysis perspective. As explained in relation to the simulation results presented in Sections V.A and V.B, the value of the third design parameter, namely the higher cutoff frequency, has to be chosen in relation to the numerical dispersion properties of the employed scheme.

Since direct extension to multipole, plane-wave, and further spatially distributed exci- 
tation forms ${ }^{41}$ is straightforward, the simple monopole model, as formulated in the present study, is directly applicable in FDTD grid excitation for a wide variety of acoustic applications. Amongst more elaborate future extensions, the formulation of bi-directional coupling between the source and the medium is of interest, in particular with regard to the study of room-loudspeaker interactions.

\section{Acknowledgements}

The authors would like to thank Mark Avis for insightful discussions on electro-acoustic sound generation, and Jonathan Hargreaves for his helpful comments on the manuscript.

\section{References}

1 J. G. Tolan and J. B. Schneider, "Locally conformal method for acoustic finite-difference time-domain modeling of rigid surfaces", The Journal of the Acoustical Society of America 114, 2575-2581 (2003).

${ }^{2}$ K. Kowalczyk and M. van Walstijn, "Modeling frequency-dependent boundaries as digital impedance filters in FDTD and K-DWM room acoustics simulations", Journal of the Audio Engineering Society 56, 569-583 (2008).

3 J. Häggblad and B. Engquist, "Consistent modeling of boundaries in acoustic finitedifference time-domain simulations", The Journal of the Acoustical Society of America 132, 1303-1310 (2012).

${ }^{4}$ S. Bilbao, "Modeling of complex geometries and boundary conditions in finite difference/finite volume time domain room acoustics simulation", IEEE Transactions on Audio, Speech, and Language Processing (2013).

${ }^{5}$ K. Kowalczyk and M. van Walstijn, "Room acoustics simulation using 3-D compact explicit FDTD schemes", IEEE Transactions on Audio, Speech, and Language Processing 19, 34-46 (2011). 
6 S. Bilbao, "Optimized FDTD schemes for 3D acoustic wave propagation", IEEE Transactions on Audio, Speech, and Language Processing (2012).

7 L. Savioja, "Real-time 3D finite-difference time-domain simulation of low-and midfrequency room acoustics", in 13th Int. Conf on Digital Audio Effects (2010).

8 J. Sheaffer and B. Fazenda, "FDTD/K-DWM simulation of 3D room acoustics on general purpose graphics hardware", in Proc. of the Institute of Acoustics, volume 32 (2010).

9 C. Webb and S. Bilbao, "Computing room acoustics with CUDA - 3D FDTD schemes with boundary losses and viscosity", in Proc. IEEE Int. Conf. on Acoustics, Speech and Sig. Proc. (Prague) (2011).

10 K. Yee, "Numerical solution of initial boundary value problems involving maxwell's equations in isotropic media", IEEE Transactions on Antennas and Propagation 14, 302-307 (1966).

11 A. Taflove and S. Hagness, Computational electrodynamics, pp. 175-224 (Artech House, Boston, Ma.) (2000).

12 S. Gedney, Introduction to the Finite-difference Time-domain (FDTD) Method for Electromagnetics, pp. 75-99 (Morgan \& Claypool Publishers, San Rafael, Ca.) (2011).

13 D. Botteldooren, "Finite-difference time-domain simulation of low-frequency room acoustic problems", The Journal of the Acoustical Society of America 98, 3302 (1995).

14 M. Karjalainen and C. Erkut, "Digital waveguides versus finite difference structures: Equivalence and mixed modeling", EURASIP Journal on Applied Signal Processing 978$989(2004)$.

15 H. Hacihabiboglu, B. Gunel, and A. Kondoz, "Time-domain simulation of directive sources in 3-D digital waveguide mesh-based acoustical models", IEEE Transactions on Audio, Speech, and Language Processing 16, 934-946 (2008).

16 T. Lokki, A. Southern, S. Siltanen, and L. Savioja, "Acoustics of epidaurus studies with room acoustics modelling methods", Acta Acustica united with Acustica 99, 40-47 (2013).

17 D. Buechler, D. Roper, C. Durney, and D. Christensen, "Modeling sources in the FDTD 
formulation and their use in quantifying source and boundary condition errors", IEEE Transactions on Microwave Theory and Techniques 43, 810-814 (1995).

18 J. Schneider, C. Wagner, and O. Ramahi, "Implementation of transparent sources in FDTD simulations", IEEE Transactions on Antennas and Propagation 46, 1159-1168 (1998).

19 J. Schneider, C. Wagner, and S. Broschat, "Implementation of transparent sources embedded in acoustic finite-difference time-domain grids", The Journal of the Acoustical Society of America 103, 136 (1998).

20 J. Redondo, R. Picó, B. Roig, and M. Avis, "Time domain simulation of sound diffusers using finite-difference schemes", Acta acustica united with acustica 93, 611-622 (2007).

21 H. Jeong and Y. Lam, "Source implementation to eliminate low-frequency artifacts in finite difference time domain room acoustic simulation", The Journal of the Acoustical Society of America 131, 258-268 (2012).

22 P. Morse and K. Ingard, Theoretical acoustics, p. 241 (McGraw-Hill, New York) (1986).

23 J. Strikwerda, Finite difference schemes and partial differential equations, p. 34 (SIAM, Philadelphia, Pa.) (2004).

24 J. Botts and L. Savioja, "Integrating finite difference schemes for scalar and vector wave equations", in IEEE Int. Conf. Acoust., Speech, Signal Processing (2013).

25 M. R. Schroeder, "Integrated-impulse method measuring sound decay without using impulses", The Journal of the Acoustical Society of America 66, 497 (1979).

26 S. Müller and P. Massarani, "Transfer-function measurement with sweeps", Journal of the Audio Engineering Society 49, 443-471 (2001).

27 R. San Martín and M. Arana, "Uncertainties caused by source directivity in room-acoustic investigations", The Journal of the Acoustical Society of America 123, EL133-EL138 (2008).

28 (Ref. 22, p. 310).

29 A. Southern, D. Murphy, T. Lokki, and L. Savioja, "The perceptual effects of dispersion error on room acoustic model auralization", in Proc. Forum Acusticum, Aalborg, 
Denmark, 1553-1558 (2011).

30 J. Botts, A. Bockman, and N. Xiang, "On the selection and implementation of sources for finite-difference methods", in Proceedings of 20th International Congress on Acoustics (2010).

31 T. Su, W. Yu, and R. Mittra, "A new look at FDTD excitation sources", Microwave and optical technology letters 45, 203-207 (2005).

32 N. Ricker, "Wavelet contraction, wavelet expansion, and the control of seismic resolution", Geophysics 18, 769-792 (1953).

33 X. Yuan, D. Borup, J. Wiskin, M. Berggren, and S. Johnson, "Simulation of acoustic wave propagation in dispersive media with relaxation losses by using FDTD method with PML absorbing boundary condition", IEEE Transactions on Ultrasonics, Ferroelectrics and Frequency Control 46, 14-23 (1999).

34 J. Tolan and J. Schneider, "Locally conformal method for acoustic finite-difference timedomain modeling of rigid surfaces", The Journal of the Acoustical Society of America $114,2575(2003)$.

35 O. Herrmann, "On the approximation problem in nonrecursive digital filter design", IEEE Transactions on Circuit Theory 18, 411-413 (1971).

36 I. Khan and R. Ohba, "Explicit formulas for coefficients of maximally flat FIR low/highpass digital filters", Electronics Letters 36, 1918-1919 (2000).

37 J. Smith, Introduction to Digital Filters: with Audio Applications, p. 272 (W3K Publishing, Stanford, Ca.) (2008).

38 (Ref. 22, p. 315).

39 R. J. Matheson, "Multichannel low frequency room simulation with properly modeled source terms-multiple equalization comparison", in Audio Engineering Society Convention 125 (2008).

40 J. Sheaffer and M. van Walstijn, "Source modelling toolbox: WWW document and computer code", (2013), URL http://code.soundsoftware.ac.uk/projects/smt/.

41 J. Escolano, J. J. López, and B. Pueo, "Directive sources in acoustic discrete-time domain 
simulations based on directivity diagrams", The Journal of the Acoustical Society of America 121, EL256-EL262 (2007). 
TABLE I. Generalization of source models using the cascaded filters approach. Inactive gains or filter blocks are indicated with a unity multiplier.

\begin{tabular}{lcccc}
\hline \hline & $H_{\mathrm{m}}(z)$ & $g_{0}$ & $H_{\mathrm{i}}(z)$ & $g_{1}$ \\
\hline \hline HS & 1 & 1 & 1 & 0 \\
SS & 1 & 1 & 1 & 1 \\
DSS & 1 & $\frac{1}{2 A_{\mathrm{s}}} \rho_{0} c X$ & $z-z^{-1}$ & 1 \\
TS & 1 & 1 & $1-I(z)$ & 1 \\
PCS (Yee) & Eq. (28) $\frac{1}{X^{2}} z_{0} \lambda A_{\mathrm{s}}$ & 1 & 1 \\
PCS (Wave) Eq. (28) $\frac{1}{X} \lambda^{2} \rho_{0} A_{\mathrm{s}} \frac{1}{2 T}\left(z-z^{-1}\right)$ & 1 \\
\hline
\end{tabular}




\section{List of Figures}

FIG. 1 Pulse spectra. (a) Gaussian (G), Blackman-Harris (BH), and Maximally Flat (MF) FIR pulse. (b) Differentiated Gaussian (DG), Sine-Modulated Gaussian (MG), and Ricker Wavelet (RW). The modulation frequency for the MG pulse and the peak frequency of the RW pulse were chosen equal to the cutoff frequeny $f_{c}=0.1 f_{s} \ldots \ldots \ldots \ldots \ldots$

FIG. 2 Unified representation of source models. $H_{\mathrm{p}}(z)$ pulse-shaping filter, $H_{\mathrm{m}}(z)$ mechanical filter, $H_{\mathrm{i}}(z)$ injection filter, $\left.s_{\mathrm{p}}\right|^{n}$ excitation signal, $\left.s_{\mathrm{g}}\right|_{\mathrm{i}^{\prime}} ^{n}$ final grid signal to be injected. . . . . . . . . . . . . . . . . . . .

FIG. 3 Sound pressure at the receiving position of a domain excited using the PCS method. (a) Time domain comparison: Yee and Wave equation (WE) methods plotted against the closed-form solution $(\mathrm{CF})$. (b) Frequency spectra: wave equation method solved with different values of $\mathcal{Q} \ldots \ldots \ldots \ldots$

FIG. 4 Calculated frequency response for three different source models, HS - hard source (response similar to TS), DSS - differentiated soft source (response similar to SS in Yee methods), PCS - physically constrained source. Excitation signals are MF FIR pulses of $N=16$ and $f_{c}=0.25 f_{s}$. PCS resonance

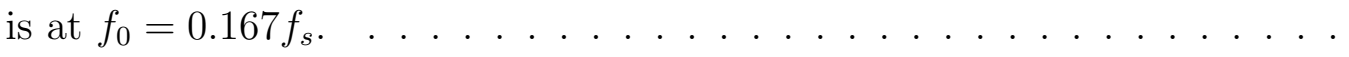

FIG. 5 Pressure at the receiving position of a grid excited by (a) hard-source, (b) differentiated soft-source and (c) physically-constrained source, at three different sample rates. . . . . . . . . . . . . . . . . .

FIG. 6 Sound pressure at the receiving position for a grid excited by a physically constrained source (PCS) compared to (a) SS - undifferentiated soft source and, (b) HS - hard source. All source models employ a Gaussian pulse shaping filter $\left(\sigma=3 \frac{1}{3} \cdot 10^{-4}\right)$. Results are normalized for visual clarity. . . . . . . 26 
FIG. 7 Source function (dashed lines), $\psi(t)$, and rate of fluid emergence (solid lines), $q(t)$ at the source node, for (a) undifferentiated soft source and (b) physically constrained source, both excited using a Gaussian pulse. Results have been normalized to $\pm 1 \mathrm{~Pa}$. and $\pm 1 \mathrm{kgm}^{-3} \mathrm{~s}^{-1}$ for visual clarity. . . . . . . . . 27 